\title{
Thirteen Camellia chloroplast genome sequences determined by high-throughput sequencing: genome structure and phylogenetic relationships
}

\author{
Hui Huang ${ }^{1}$, Chao Shi ${ }^{1,2}$, Yuan Liu ${ }^{1,2}$, Shu-Yan Mao ${ }^{1}$ and Li-Zhi Gao ${ }^{1 *}$
}

\begin{abstract}
Background: Camellia is an economically and phylogenetically important genus in the family Theaceae. Owing to numerous hybridization and polyploidization, it is taxonomically and phylogenetically ranked as one of the most challengingly difficult taxa in plants. Sequence comparisons of chloroplast (cp) genomes are of great interest to provide a robust evidence for taxonomic studies, species identification and understanding mechanisms that underlie the evolution of the Camellia species.

Results: The eight complete $\mathrm{cp}$ genomes and five draft cp genome sequences of Camellia species were determined using Illumina sequencing technology via a combined strategy of de novo and reference-guided assembly. The Camellia cp genomes exhibited typical circular structure that was rather conserved in genomic structure and the synteny of gene order. Differences of repeat sequences, simple sequence repeats, indels and substitutions were further examined among five complete $\mathrm{cp}$ genomes, representing a wide phylogenetic diversity in the genus. A total of fifteen molecular markers were identified with more than $1.5 \%$ sequence divergence that may be useful for further phylogenetic analysis and species identification of Camellia. Our results showed that, rather than functional constrains, it is the regional constraints that strongly affect sequence evolution of the cp genomes. In a substantial improvement over prior studies, evolutionary relationships of the section Thea were determined on basis of phylogenomic analyses of $\mathrm{cp}$ genome sequences.
\end{abstract}

Conclusions: Despite a high degree of conservation between the Camellia cp genomes, sequence variation among species could still be detected, representing a wide phylogenetic diversity in the genus. Furthermore, phylogenomic analysis was conducted using 18 complete $\mathrm{cp}$ genomes and 5 draft $\mathrm{cp}$ genome sequences of Camellia species. Our results support Chang's taxonomical treatment that C. pubicosta may be classified into sect. Thea, and indicate that taxonomical value of the number of ovaries should be reconsidered when classifying the Camellia species. The availability of these cp genomes provides valuable genetic information for accurately identifying species, clarifying taxonomy and reconstructing the phylogeny of the genus Camellia.

Keywords: Camellia, Chloroplast genome, Phylogenetic relationships, Genomic structure, Taxonomic identification

\section{Background}

Camellia, comprising more than 200 species, is an economically and phylogenetically important genus in the family Theaceae [1]. Besides the abundance in phenotypic and species diversity, increasing attention has been paid to the genus, as they include several

\footnotetext{
* Correspondence: Lgao@mail.kib.ac.cn

'Plant Germplasm and Genomics Center, Germplasm Bank of Wild Species in Southwest China, Kunming Institute of Botany, Chinese Academy of

Sciences, Kunming 650201, China

Full list of author information is available at the end of the article
}

economically important members of their commercial and ornamental values. One of the most economic values of Camellia is the production of tea made from the young leaves of $C$. sinensis var. sinensis and $C$. sinensis var. assamica in the section Thea. The other most economically important species is $C$. oleifera, which has the longest history of cultivation and utilization in China for edible oil used primarily in cooking. Many other species of the genus Camellia were also used locally for seed oil production, such as C. reticulata [2]. Moreover, the Camellia species are of great ornamental values, 
particularly represented by $C$. japonica, C. reticulata and C. sasanqua.

As a result of frequent hybridization and polyploidization, Camellia is taxonomically and phylogenetically regarded as one of the most challengingly difficult taxa in plants. Traditional classification of species using a morphology-based system is often dynamic and unreliable, which is often affected by environmental factors. The lack of suitable DNA fragments and polymorphic genetic markers for phylogenic analysis have long obstructed the availability of a reliable phylogeny, adding the controversies about taxonomic classification that prevent us from better understanding the diversification and evolution of the genus Camellia. By using amplified fragment length polymorphisms (AFLPs) [3], simple sequence repeats (SSRs) [4], random amplified polymorphic DNA (RAPD) [5], inter-simple sequence repeat (ISSR) [6], internal transcribed spacer (ITS) [1,7] and several DNA loci [8], a number of previous studies gave further insights into the taxonomy and phylogeny of the Camellia species but still have not reached a satisfied resolution. A recent effort using whole chloroplast (cp) genome sequences of six Camellia species has generated useful data but still failed to determine their phylogenetic relationships, not agreeing with any taxonomic treatments [9].

The cp genomes could provide valuable information for taxonomic classification and the reconstruction of phylogeny as a result of sequence divergence between plant species and individuals. Owing to the absence of recombination and maternal transmission, the $\mathrm{cp}$ genomes are helpful for tracing source populations [10,11] and phylogenetic studies of higher plants for resolving complex evolutionary relationships [12-14]. It is particularly true for the case of Camellia, given its confusing phylogenetic relationships with large nuclear genomes [15]. Cp-derived markers, e.g. rpl16 gene, psbA-trnH, trnL-F and rpl32-trnL intergenic spacer (IGS), were employed to study evolutionary relationships between tea plants $[8,16]$. Repetitive sequences within the cp genomes are also potentially useful for ecological and evolutionary studies of plants [17]. Not only will the information from cp genomes be useful for studying the taxonomy and phylogenetic relationships, but it will also facilitate $\mathrm{cp}$ transformation in the economically important plants. The next-generation sequencing techniques have revolutionized DNA sequencing via high-throughput capabilities but relatively low costs. As it is now more convenient to obtain cp genome sequences and promptly extend gene-based phylogenetics to phylogenomics.

In this study, we sequenced the 13 Camellia chloroplast genomes using next-generation Illumina genome analyzer platform. The sequenced Camellia species included up to 10 species and varieties (10/18) from sect. Thea with an emphasis of these species belonging to the section. Three representative species were additionally sampled, each from sect. Camellia, sect. Corallina and sect. Archecamellia, respectively. This study aims to examine global patterns of structural variation of the Camellia cp genomes and reconstruct phylogenetic relationships among the representative species. The complete cp genome sequences of Camellia reported here are prerequisite for classifying the 'difficult taxa' and modifying these important economic plants by chloroplast genetic engineering techniques.

\section{Results and discussion}

\section{Chloroplast genome sequencing and assembly}

Using Illumina genome analyzer platform, we sequenced cp genomes of seven species and three varieties from sect. Thea, and each from sect. Archecamellia, Corallina and Camellia, respectively (Table 1). The three cp genomes of $C$. sinensis var. assamica, C. oleifera and $C$. taliensis [18] were used in our study with a minor revision by the two following steps: 1) assembled and manually checked more carefully; 2) PCR were used with high fidelity polymerase to verify the sequences in the four junctions. And then the revised $C$. sinensis var. assamica was employed as a reference while assembling the 13 sequenced $\mathrm{cp}$ genomes. Illumina paired-end $(2 \times 100 \mathrm{bp})$ sequencing produced large data sets for individual species. 5,504,058 (C. tachangensis) to 111,673,521 (C. sinensis var. sinensis) paired-end reads were mapped to the reference cp genome of $C$. sinensis var. assamica,

Table 1 Information of the sequenced Camellia chloroplast genomes according Min's taxonomic treatment [2]

\begin{tabular}{|c|c|c|c|}
\hline Species & Subgenus & Section & $\begin{array}{l}\text { Collection } \\
\text { sites }\end{array}$ \\
\hline $\begin{array}{l}\text { Camellia crassicolumna var. } \\
\text { crassicolumna }\end{array}$ & Thea & Thea & TRI \\
\hline Camellia fangchengensis & Thea & Thea & ICSG \\
\hline Camellia grandibracteata & Thea & Thea & $\mathrm{TRI}$ \\
\hline Camellia kwangsiensis & Thea & Thea & $\mathrm{TRI}$ \\
\hline Camellia leptophylla & Thea & Thea & ICSG \\
\hline Camellia ptilophylla & Thea & Thea & ICSG \\
\hline $\begin{array}{l}\text { Camellia sinensis var. } \\
\text { dehungensis }\end{array}$ & Thea & Thea & TRI \\
\hline Camellia sinensis var. sinensis & Thea & Thea & $\mathrm{TRI}$ \\
\hline Camellia sinensis var. pubilimba & Thea & Thea & TRI \\
\hline Camellia tachangensis & Thea & Thea & TRI \\
\hline Camellia petelotii & Thea & Archecamellia & ICSG \\
\hline Camellia pubicosta & Thea & Corallina & ICSG \\
\hline Camellia reticulata & Camellia & Camellia & $\mathrm{KIB}$ \\
\hline
\end{tabular}

TRI, Tea Research Institute, Yunnan Academy of Agricultural Science (Menghai, Yunnan, China);

ICSG, International Camellia Species Garden (Jinhua, Zhejiang, China); $\mathrm{KIB}$, Kunming Institute of Botany, Chinese Academy of Sciences (Kunming, Yunnan, China). 
reaching 35 to $711 \times$ coverage on average across these $\mathrm{cp}$ genomes. After de novo and reference-guided assembly as in [19] with minor modifications, we obtained eight complete cp genomes and five draft cp genomes (Table 2). The four junction regions for each resulting $\mathrm{cp}$ genome were validated by using PCR-based sequencing with four pairs of primers (Additional file 1: Table S1). These genome sequences were deposited into the GenBank under accession numbers (KJ806274-KJ806286) (Additional file 2: Table S2).

\section{Conservation of Camellia chloroplast genomes}

All eight complete Camellia cp genomes were composed of single circular double-stranded DNA molecules. They displayed the typical quadripartite structure of most angiosperms, including the large single copy (LSC), the small single copy (SSC) and a pair of inverted repeats (IRa and IRb). There were no obvious sequence inversions or genomic rearrangements (Figure 1). Among these cp genomes, genome size ranged from 156,971 bp (C. reticulata) to 157,127 bp (C. grandibracteata). The length varied from $86,606 \mathrm{bp}$ (C. reticulata) to 86,679 bp (C. sinensis var. pubilimba) in the LSC region, from 18,235 bp (C. reticulata) to $18,286 \mathrm{bp}$ (C. grandibracteata) in the SSC region, and from $26,065 \mathrm{bp}$ (C. reticulata) to $26,096 \mathrm{bp}$ (C. sinensis var. pubilimba) in IR region. Each cp genome was found to harbor a total of 131 genes, including 86 protein-coding genes, 37 transfer RNA (tRNA) genes, and eight ribosomal RNA (rRNA) genes (Table 3). Of them, we identified 13 protein-coding genes, 14 tRNA coding genes and eight rRNA coding genes that are located within IRs. The LSC region contained 61 protein-coding and 22 tRNA genes, while the SSC region had 11 protein-coding and one tRNA gene. The rps12 gene is an uniquely divided gene with the $5^{\prime}$ end exon located in the LSC region while two copies of $3^{\prime}$ end exon and intron are located in the IRs. The $y c f 1$ is located in the boundary regions between IRa/SSC, leading to incomplete duplication of the gene within IRs. There were 18 intron-containing genes, including six tRNA genes and 12 protein-coding genes, almost all of which are single-intron genes except for $y c f 3$ and $c l p P$, each having two introns (Table 3). matK was located within the intron of trnK-UUU with the largest intron $(2,487 \mathrm{bp})$. It was found that $y c f 1, a c c D$, rpl23 and $y c f 2$ are often absent in plants [20], but they were detected in the reported Camellia cp genomes in this study. Similar to other higher plants, one pair of genes, atpB-atpE, was observed to overlap each other with 3 -bp. However, $p s b C$ - $p s b D$ had a 52-bp overlapping region in the Camellia cp genomes, which was observed an overlapping 53-bp in Gossypium [21]. Note that coding and non-coding regions account for $44.6 \%$ and $55.4 \%$ of the whole cp genome, respectively. The overall GC content was approximately $37.3 \%$, which is almost identical with each other among the eight complete Camellia cp genomes.

Table 2 The sequenced chloroplast genome features

\begin{tabular}{|c|c|c|c|c|c|c|c|}
\hline Complete genomes & $\begin{array}{l}\text { Matched } \\
\text { reads (bp) }\end{array}$ & $\begin{array}{l}\text { Genome } \\
\text { size (bp) }\end{array}$ & $\begin{array}{l}\text { Mean } \\
\text { coverage }\end{array}$ & LSC length (bp) & SSC length (bp) & IR length (bp) & GC content $(\%)$ \\
\hline C. grandibracteata & $24,127,775$ & 157,127 & 154 & 86,657 & 18,286 & 26,092 & 37.29 \\
\hline C. leptophylla & $26,635,918$ & 157,102 & 170 & 86,648 & 18,276 & 26,089 & 37.30 \\
\hline C. sinensis var. dehungensis & $24,978,790$ & 157,110 & 159 & 86,656 & 18,276 & 26,089 & 37.30 \\
\hline C. sinensis var. sinensis & $111,673,521$ & 157,117 & 711 & 86,663 & 18,276 & 26,089 & 37.29 \\
\hline C. sinensis var. pubilimba & $7,753,104$ & 157,086 & 49 & 86,679 & 18,267 & 26,096 & 37.30 \\
\hline C. petelotii & $9,358,318$ & 157,121 & 60 & 86,660 & 18,283 & 26,089 & 37.29 \\
\hline C. pubicosta & $36,142,305$ & 157,076 & 230 & 86,650 & 18,280 & 26,073 & 37.30 \\
\hline C. reticulata & $56,357,778$ & 156,971 & 359 & 86,606 & 18,235 & 26,065 & 37.30 \\
\hline C. oleifera & $8,162,492$ & 157,145 & 52 & 86,676 & 18,291 & 26,089 & 37.28 \\
\hline C. sinensis var. assamica & $2,828,916$ & 157,121 & 18 & 86,651 & 18,286 & 26,092 & 37.29 \\
\hline C. taliensis & $2,828,754$ & 157,087 & 18 & 86,650 & 18,287 & 26,075 & 37.29 \\
\hline Incomplete genomes & $\begin{array}{l}\text { Matched } \\
\text { reads (bp) }\end{array}$ & $\begin{array}{l}\text { Predicted } \\
\text { genome } \\
\text { size (bp) }\end{array}$ & $\begin{array}{l}\text { Mean } \\
\text { coverage }\end{array}$ & $\begin{array}{l}\text { Number } \\
\text { of gaps }\end{array}$ & Gap length (bp) & & \\
\hline C. crassicolumna var. crassicolumna & $6,595,133$ & 157,100 & 42 & 180 & 40,530 & & \\
\hline C. fangchengensis & $39,446,507$ & 157,364 & 251 & 138 & 30,491 & & \\
\hline C. kwangsiensis & $8,553,876$ & 156,992 & 54 & 207 & 36,424 & & \\
\hline C. ptilophylla & $8,426,943$ & 157,057 & 54 & 148 & 22,817 & & \\
\hline C. tachangensis & $5,504,058$ & 157,009 & 35 & 345 & 57,250 & & \\
\hline
\end{tabular}




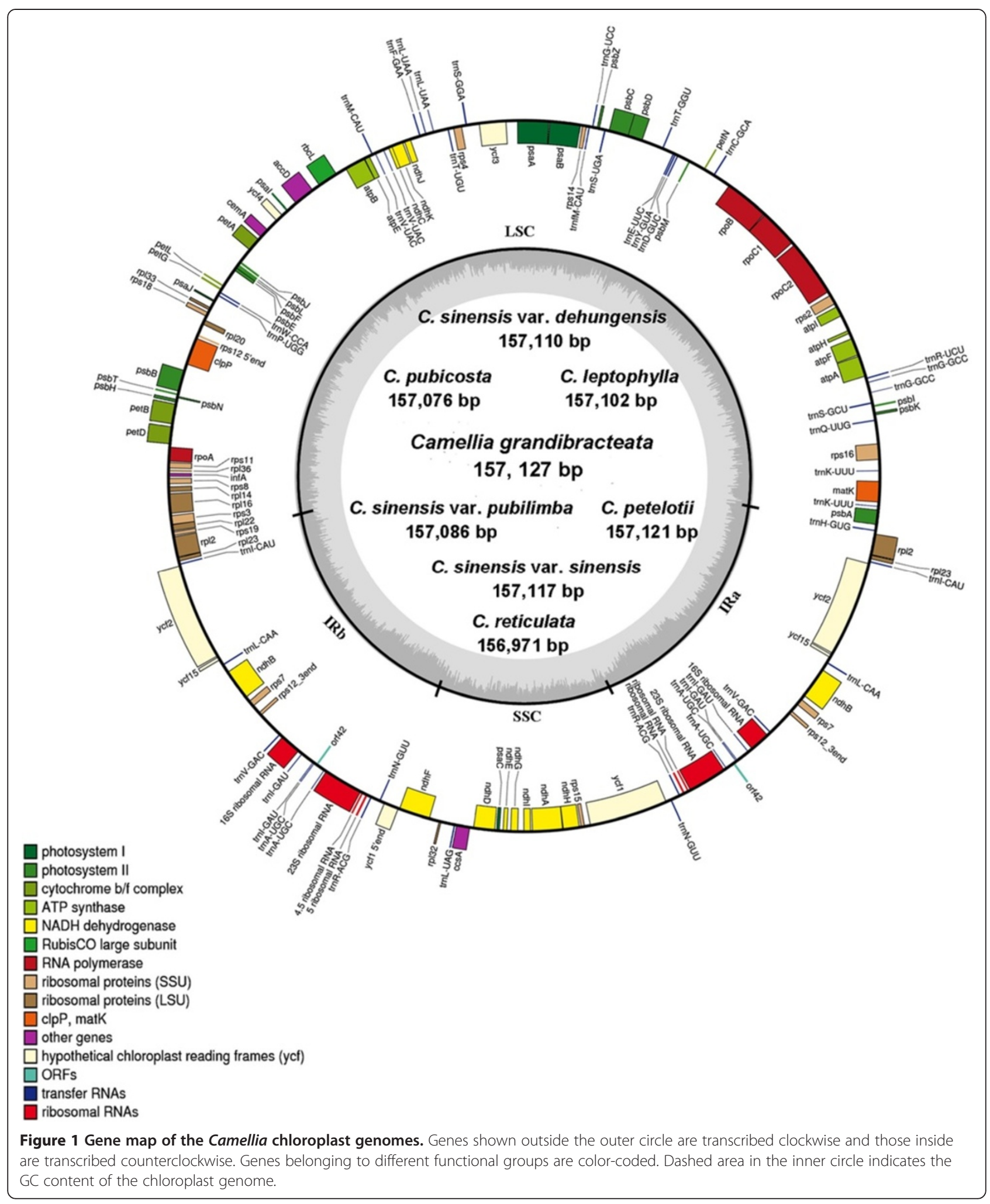

Although genome size and overall genomic structure including gene number and gene order are well conserved, IR expansion/contraction is common in plant cp genomes. In grasses, for example, the termini of two genes, $n d h H$ and $n d h F$, were reported to have repeatedly migrated into and out of the adjacent IRs [22]. Whole rps19 was located within the LSC region in the most Gossypium cp genomes but failed to find in cp genome of G. raimondii D5 [21]. 
Table 3 Genes contained in the sequenced Camellia chloroplast genomes

\begin{tabular}{|c|c|c|}
\hline Category & Group of genes & Name of genes \\
\hline \multirow[t]{5}{*}{ Self replication } & $\begin{array}{l}\text { Large subunit of } \\
\text { ribosomal proteins }\end{array}$ & $r p \mid 2^{b, c}, 14,16^{b}, 20,22,23^{c}, 32,33,36$ \\
\hline & $\begin{array}{l}\text { Small subunit of } \\
\text { ribosomal proteins }\end{array}$ & $\begin{array}{l}\operatorname{rps} 2,3,4,7^{c}, 8,11,12^{b-d}, 14,15 \\
16^{b}, 18,19\end{array}$ \\
\hline & $\begin{array}{l}\text { DNA dependent } \\
\text { RNA polymerase }\end{array}$ & $\operatorname{rpo} A, B, C 1^{b}, C 2$ \\
\hline & rRNA genes & $\operatorname{rrn} 4.5^{c}, 5^{c}, 16^{c}, 23^{c}$ \\
\hline & tRNA genes & 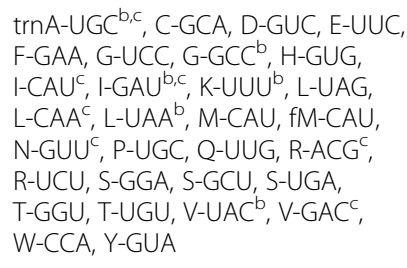 \\
\hline \multirow[t]{6}{*}{ Photosynthesis } & Photosystem I & psaA, B, C, I, J, ycf3 ${ }^{a}, y c f 4$ \\
\hline & Photosystem II & $\operatorname{psbA}, B, C, D, E, F, H, I, J, K, L, M, N, T, Z$ \\
\hline & $\begin{array}{l}\mathrm{NADH} \\
\text { oxidoreductase }\end{array}$ & $n d h A^{b}, B^{b, c}, C, D, E, F, G, H, I, J, K$ \\
\hline & $\begin{array}{l}\text { Cytochrome b6/f } \\
\text { complex }\end{array}$ & $\operatorname{pet} A, B^{b}, D^{b}, G, L, N$ \\
\hline & ATP synthase & $\operatorname{atp} A, B, E, F^{b}, H, I$ \\
\hline & Rubisco & $\mathrm{rbcL}$ \\
\hline \multirow[t]{6}{*}{ Other gene } & $\begin{array}{l}\text { Translational } \\
\text { initiation factor }\end{array}$ & $\operatorname{infA}$ \\
\hline & Maturase & matk \\
\hline & Protease & $\mathrm{clpP}^{\mathrm{a}}$ \\
\hline & $\begin{array}{l}\text { Envelop } \\
\text { membrane protein }\end{array}$ & cemA \\
\hline & $\begin{array}{l}\text { Subunit Acetyl- } \\
\text { CoA-carboxylase }\end{array}$ & $\mathrm{accD}$ \\
\hline & $\begin{array}{l}\text { c-type cytochrom } \\
\text { synthesis gene }\end{array}$ & $\operatorname{ccs} A$ \\
\hline $\begin{array}{l}\text { Unknown } \\
\text { gene }\end{array}$ & $\begin{array}{l}\text { Conserved Open } \\
\text { Reading Frames }\end{array}$ & ycf1, $2^{c}, 15^{c}$, orf 42 \\
\hline
\end{tabular}

${ }^{\mathrm{a}}$ Genes containing two introns;

${ }^{\mathrm{b}}$ Genes containing a single intron

'Two gene copies in the IRs;

${ }^{\mathrm{d}}$ Genes split into two independent transcription units.

Kim et al. [23] considered that the length of angiosperm $\mathrm{cp}$ genomes is variable primarily due to the expansion and contraction of the inverted repeat IR region and the single-copy boundary regions. The IR/SC boundary regions of the 18 complete Camellia cp genomes were compared, showing slight differences in junction positions (Figure 2). The junction positions were conserved across 12 Camellia cp genomes and were variable in cp genomes of C. taliensis 7, C. reticulata, C. sinensis var. pubilimba, C. danzaiensis, C. pitardii and C. impressinervis. For example, the distances from $n d h F$ and $y c f 15^{\prime}$ end to the junction of IRb/SSC were 3 and $4 \mathrm{bp}$, respectively in $C$. reticulata, and 6 and 9 bp, respectively, in C. sinensis var. pubilimba due to the deletion. The distances were 6 and 64 bp, respectively, in C. danzaiensis, C. pitardii and C. impressinervis, which is different from the distances of 57 and $13 \mathrm{bp}$, respectively, in other 12 Camellia cp genomes. The gene $y c f 1$ extended into the IRa region with $1,068 \mathrm{bp}$ in the $12 \mathrm{cp}$ genomes, with $1,048 \mathrm{bp}$ in $C$. reticulata, with 1,067 bp in C. taliensis 7, with 1,036 bp in C. sinensis var. pubilimba, and with 1,042 bp in C. danzaiensis, C. pitardii and C. impressinervis cp genomes.

To investigate levels of genome divergence, multiple alignments of 18 Camellia cp genome sequences and nine representative plants with fully sequenced $\mathrm{cp}$ genomes were performed (Figure 3 and Additional file 2: Table S2). With C. sinensis var. assamica as a reference, we plotted sequence identity using VISTA [24]. The results revealed high sequence similarity across the 18 Camellia cp genomes, suggesting that Camellia cp genomes are rather conserved. However, the marked differences were observed between Camellia cp genomes and other plants, including Coffea arabica. As expected, the IRs are more conserved than single-copy regions, and coding regions are more conserved than noncoding regions. The most divergent coding regions were $m a t K, r p o C 2$, accD, rps19, $y c f 2$ and $y c f 1$ (Figure 3). Considering high conservation of Camellia cp genomes, as above described, we only included C. sinensis var. assamica (ASSA, sect. Thea), C. oleifera (OLEI, sect. Paracamellia), C. reticulata (RETI, sect. Camellia), C. petelotii (PETE, sect. Archecamellia) and C. pubicosta (PUBI, sect. Corallina) that represent a wide phylogenetic diversity to compare and characterize their $\mathrm{cp}$ genomic structural variations (Additional file 3: Figure S1).

\section{Repetitive sequences}

Repeated sequences are generally considered to be uncommon in cp genomes with the notable exception of a large IR present in most land plants [25]. In order to avoid redundancy, repeat sequences analysis in the five Camellia cp genomes mentioned above was carried out with a single IR region. A total of 156 repeats were detected in these cp genomes using REPuter [26], including direct, reverse and palindromic repeats (Additional file 4: Table S3). Number and distribution of repeats are rather conserved between $\mathrm{cp}$ genomes of ASSA, PUBI and RETI, excluding reverse repeats. Nevertheless, OLEI and PETE cp genomes included three repeat types, that is, direct, reverse and palindromic repeats (Figure 4). Among them, direct repeats are the most common, accounting for $62 \%$ of the total repeats, followed with palindromic repeat (19\%) and reverse repeat (19\%) (Figure 4A). The lengths of repeats in these five Camellia $\mathrm{cp}$ genomes were much shorter, ranging from 30 to 82 bp (Figure 4B, C and D), whereas much longer repeats, such as 132-bp and 287-bp repeats were found in the Poaceae and Fabaceae [27-29]. Palindromic and 


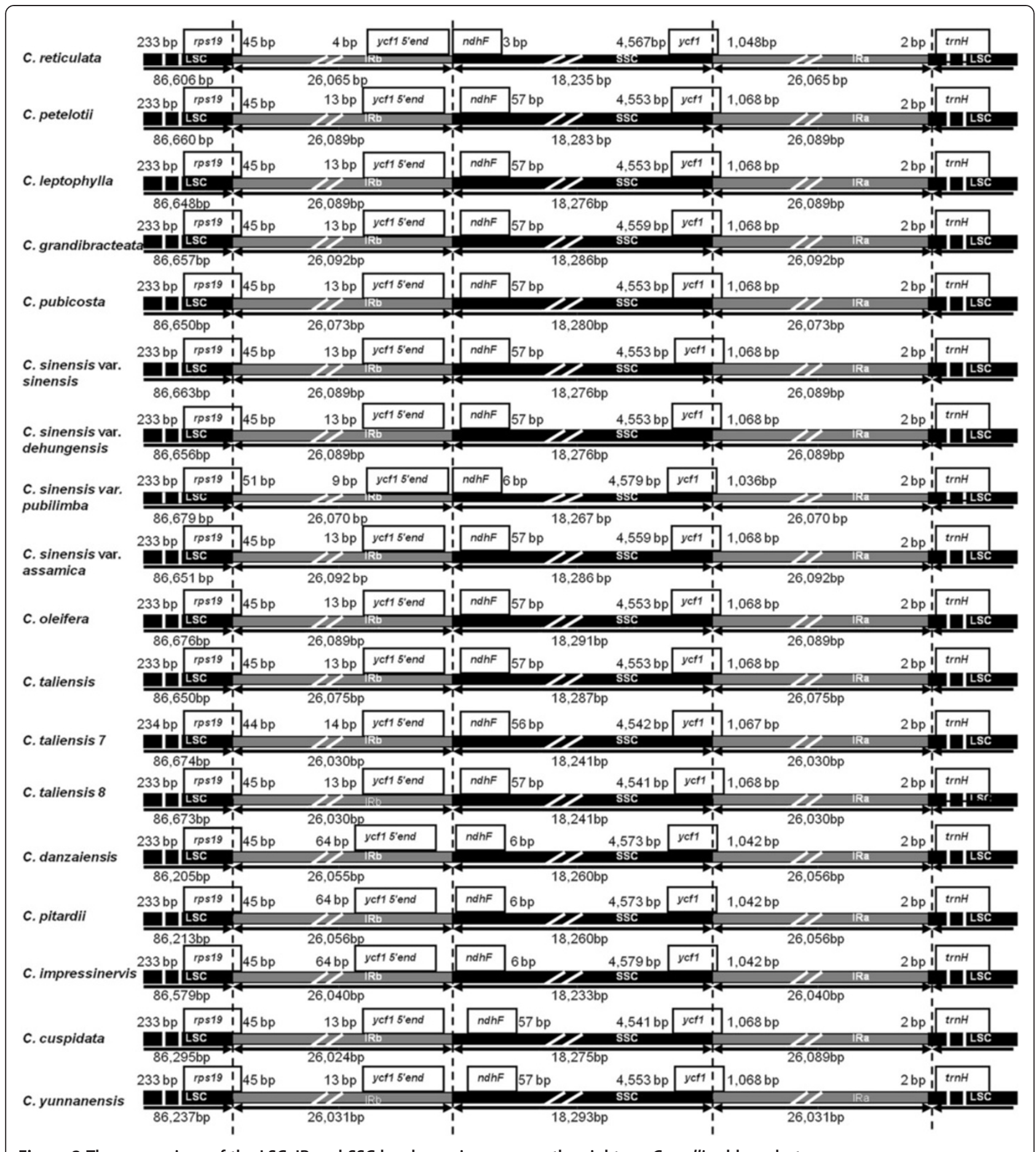

Figure 2 The comparison of the LSC, IR and SSC border regions among the eighteen Camellia chloroplast genomes.

reverse repeats occurred in a narrower size, ranging from 30-50 bp and 30-35 bp, respectively. In this study, although a minority of repeats was found in intron (6\%), the majority were located in IGS (62\%) and coding sequence (CDS) regions (32\%) (Figure 4E). Then, we investigated the repeats shared among the five Camellia cp genomes.
Here, we defined repeats that had identical lengths and located in homologous regions as shared repeats. Under such criteria there were 17 repeats shared by the five Camellia cp genomes and three repeats were presented jointly in four cP genomes. PETE had the most unique repeats (13), while ASSA, OLEI and RETI showed no 


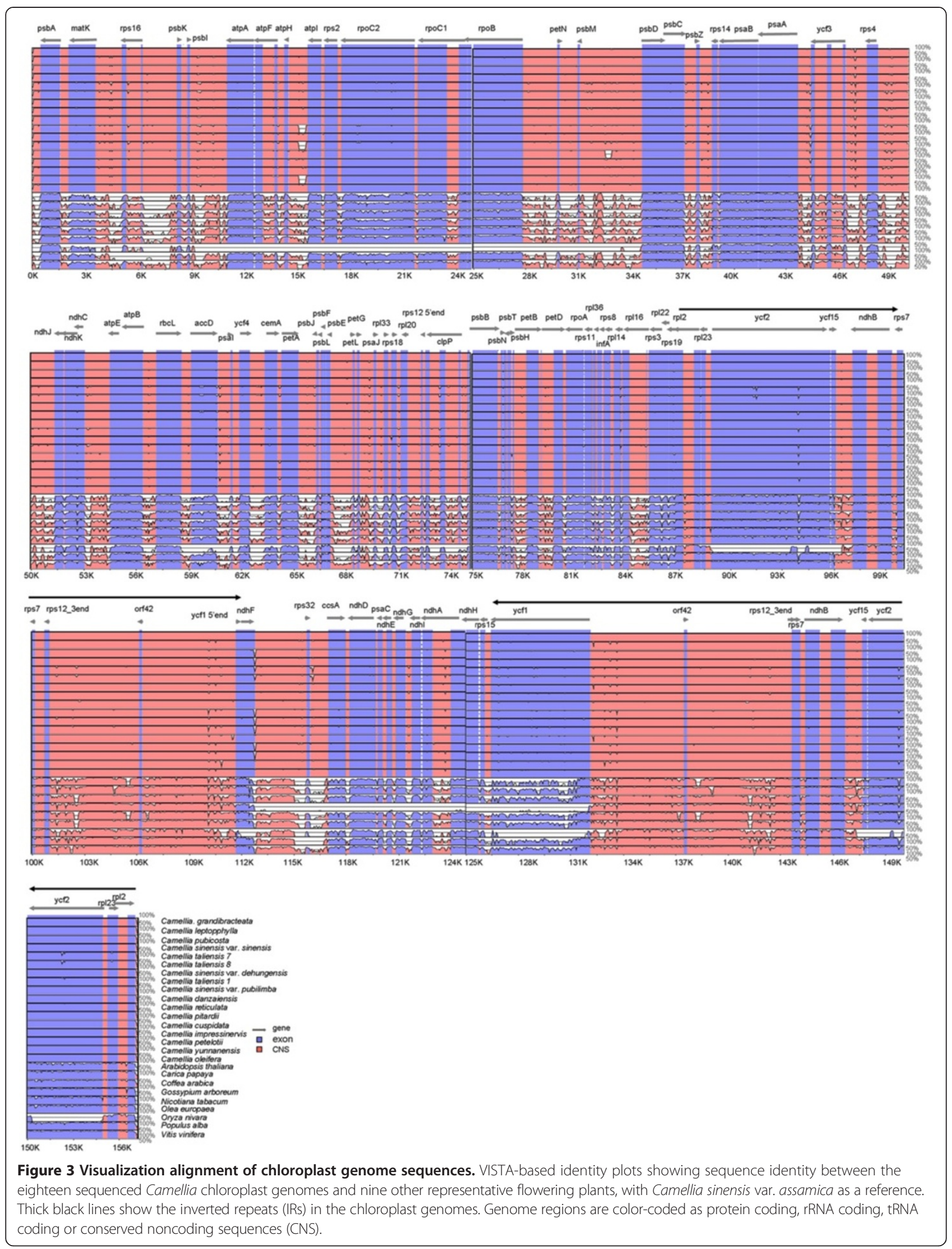




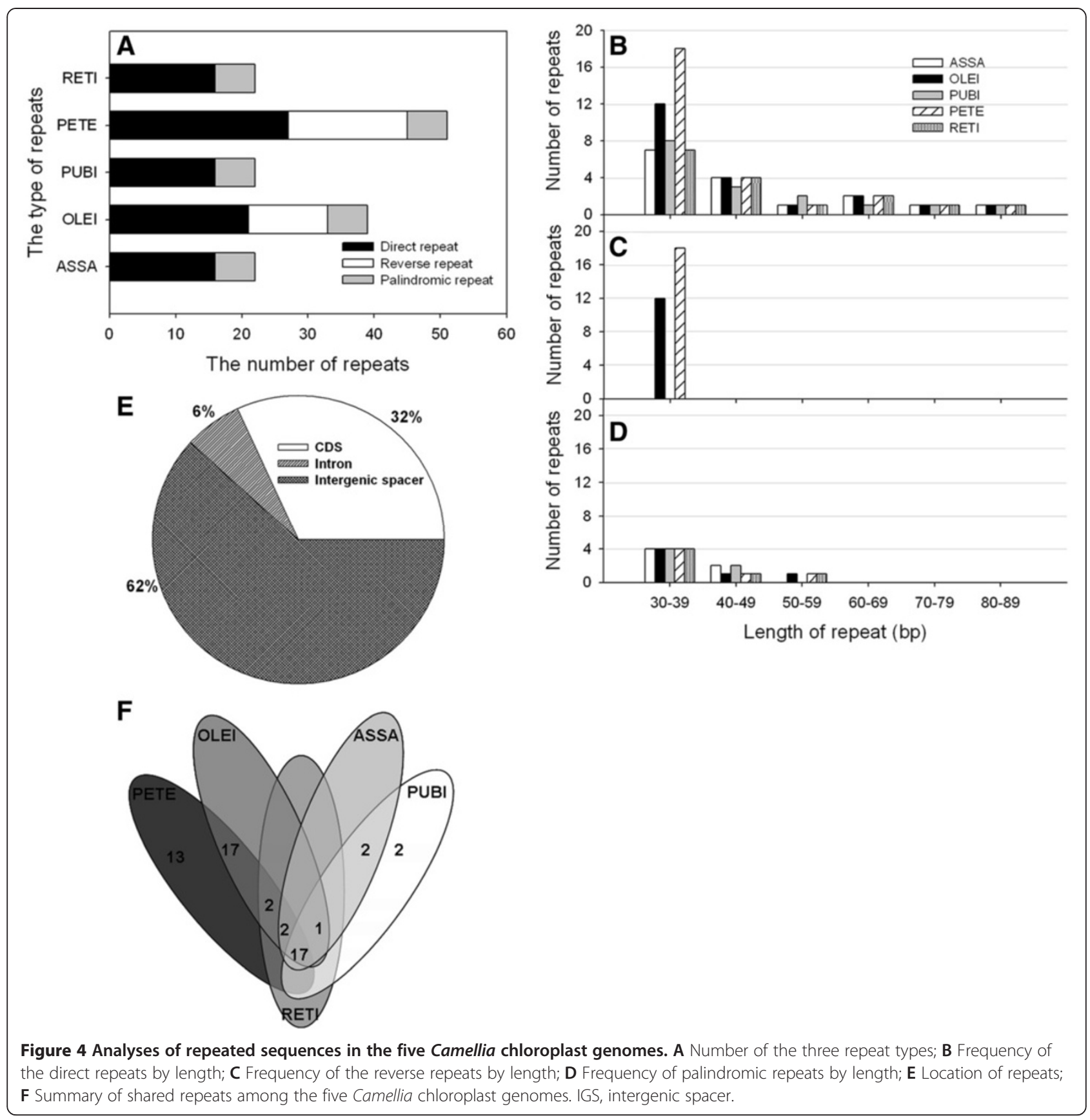

unique repeats (Figure 4F). Previous work suggested that repeat sequences have played an important role in genomic rearrangement and sequence variation in $\mathrm{cp}$ genomes through illegitimate recombination and slipped-strand mispairing [30-32]. The existence of these repeats implies that the region is a potential hotspot for genomic reconfiguration [33]. Our results also showed that divergent regions of $\mathrm{cp}$ genomes were associated with various repeat sequences such as intergenic atpF/atpH. These repeats may further serve as genetic markers for phylogenetic and population genetic studies.

\section{SSR polymorphisms}

SSRs usually have a higher mutation rate compared to other neutral DNA regions due to slipped DNA strands. They thus are often used as genetic markers, providing useful information concerning plant population genetics and ecological and evolutionary studies due to their non-recombinant, haploid and uniparentally inherited nature [34,35]. In total, 53, 51, 50, 55 and 55 SSRs were found in the cp genomes of ASSA, OLEI, PUBI, PETE and RETI, respectively (Figure 5, Table 4 and Additional file 5: Table S4). Mononucleotide 


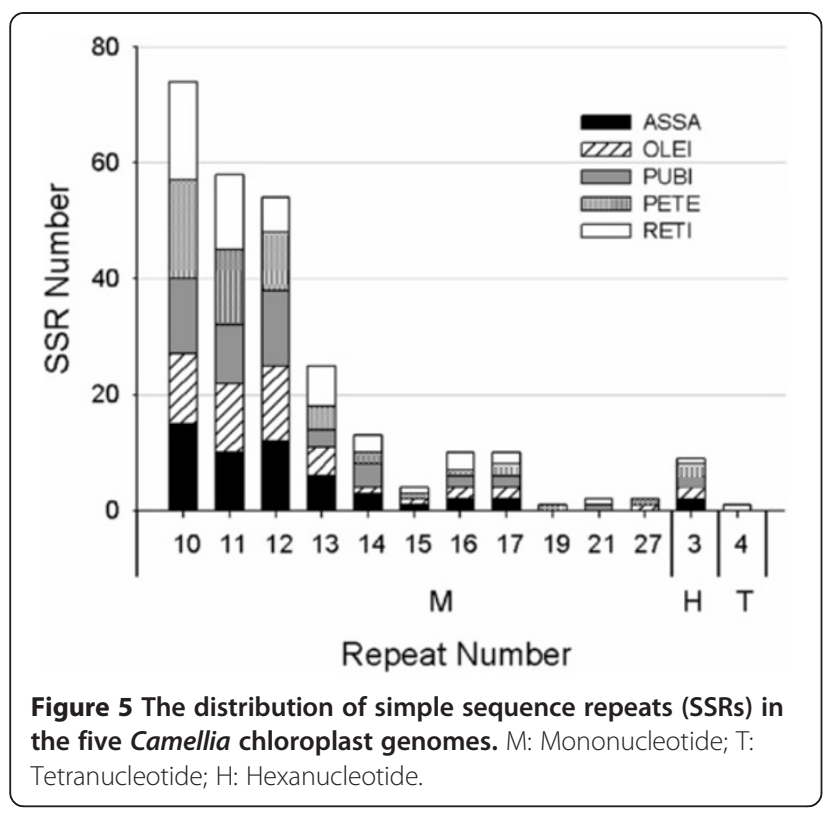

$(\mathrm{A} / \mathrm{T})$ and hexanucleotide (AAAAAG/CTTTTT) repeats were detected in the five Camellia cp genomes. One tetranucleotide (AGGG/CCCT) repeat was only found in RETI cp genome, and no dinucleotide and trinculeotide repeats were observed. The repeat unit $\mathrm{A} / \mathrm{T}$ was found to be the most abundant with particular repeat numbers of 10, 11 and 12 (Figure 5). The finding is consistent with a previous observation that $\mathrm{cP}$ SSRs were dominated by A or $\mathrm{T}$ mononucleotide repeats [36]. Mononucleotide and hexanucleotide repeats were composed of $\mathrm{A}$ or $\mathrm{T}$ at a higher level, which reflects a biased base composition with an overall A-T richness in the cp genomes [28,37]. Within the five Camellia cp genomes, SSR loci mainly located in IGS, following by CDS and introns. There were A/T (12) SSRs located in CDS-IGS (psbI, psbI/trnS-GCU) expect for PETE. No SSRs were found in the tRNAs and rRNAs. We observed that 11 SSRs located in seven protein-coding genes $[y c f 1(\times 5), \operatorname{ccs} A, \operatorname{rpoB}, \operatorname{atp} B$, rpoA, rрoC2, ndhK] of the five Camellia cp genomes. Jakobsson et al. [38] indicated that cp SSRs located in the non-coding regions of the $\mathrm{cp}$ genome commonly show intraspecific variation in repeat number. Most of those SSRs loci were located in LSC region, followed by IR and SSC regions. We found that 11 SSR loci were located in IRs of the five Camellia cp genomes. This observation is surprising because concerted evolution, as suggested earlier, should lead to exact sequence duplication in IRa compared with IRb, and therefore both IRs should contain the same number of nucleotide repeats. This may be explained by the incomplete repeat of $y c f 1$ in IRb that led to the five SSRs located in IRb and inexactly identical SSRs between IRa and IRb. Length variations in SSRs have served as useful markers for identifying crop varieties and performing population genetic studies $[39,40]$. cp SSRs characterized in this study could undoubtedly provide an assay for detecting polymorphisms at the population-level and comparing more distantly phylogenetic relationships at the genus level or above.

Table 4 Simple sequence repeats (SSRs) in the five representative Camellia chloroplast genomes

\begin{tabular}{|c|c|c|c|c|c|c|c|c|c|}
\hline \multirow[t]{2}{*}{ Genomes } & \multirow[t]{2}{*}{ Repeat units } & \multirow[t]{2}{*}{ Number } & \multicolumn{4}{|c|}{ Location } & \multicolumn{3}{|c|}{ Region } \\
\hline & & & Intron & IGS & CDS-IGS & CDS & LSC & SSC & IR \\
\hline \multicolumn{10}{|l|}{$\overline{A S S A}$} \\
\hline & $\mathrm{A} / \mathrm{T}$ & 51 & 5 & 34 & 1 & 11 & 39 & 3 & 9 \\
\hline & AAAAAG/CTITIT & 2 & & 2 & & & & & 2 \\
\hline \multicolumn{10}{|l|}{ OLEI } \\
\hline & $\mathrm{A} / \mathrm{T}$ & 49 & 6 & 31 & 1 & 11 & 36 & 4 & 9 \\
\hline & AAAAAG/CTITIT & 2 & & 2 & & & & & 2 \\
\hline \multicolumn{10}{|l|}{ PUBI } \\
\hline & $\mathrm{A} / \mathrm{T}$ & 48 & 5 & 31 & 1 & 11 & 35 & 4 & 9 \\
\hline & AAAAAG/CTTITT & 2 & & 2 & & & & & 2 \\
\hline \multicolumn{10}{|l|}{ PETE } \\
\hline & $\mathrm{A} / \mathrm{T}$ & 53 & 7 & 35 & 0 & 11 & 41 & 3 & 9 \\
\hline & AAAAAG/CTTTIT & 2 & & 2 & & & & & 2 \\
\hline \multicolumn{10}{|l|}{ RETI } \\
\hline & $\mathrm{A} / \mathrm{T}$ & 53 & 7 & 34 & 1 & 11 & 40 & 4 & 9 \\
\hline & AGGG/CCCT & 1 & & 1 & & & & & 1 \\
\hline & AAAAAG/CTTITा & 1 & & 1 & & & & & 1 \\
\hline
\end{tabular}




\section{Substitution and indel variation}

Global alignment of the five Camellia cp genomes revealed that total substitutions varied from 82 (ASSA vs. PUBI) to 265 (ASSA vs. OLEI) (Table 5). The base substitution types between $C$ and $G$ were fewer than other types, in agreement with a previous study [40]. A comparison of indels among these cp genomes (Table 5) showed that the number of indels ranged from 28 (ASSA vs. PUBI) to 72 (PUBI vs. RETI). These indel events were mainly attributed to the repetition of an adjacent sequence, probably caused by slipped-strand mis-pairing in DNA replication [41]. Indels are thought to be a major driving force in sequence evolution $[42,43]$. We observed that there were the fewest substitutions and indels between ASSA and PUBI. The ratios of nucleotide substitution events to indel events (S/I) for different pairwise comparisons showed that, among the five Camellia cp genomes, the S/I ratio varied from 2.67 (PETE vs. RETI) to 5.00 (ASSA vs. OLEI) (Table 5). Given the S/I ratio increased with divergence times between genomes [44], ASSA was inferred to be close to PUBI $(S / I=2.93)$, suggesting that PUBI may be classified into sect. Thea. The likely explanation is that the S/I ratio increased with the increase of divergence times that may arise from systematic underestimation of indels in more distantly related species [44].

The number of short indels (1-10 bp) accounted for $>90 \%$ of total indels (Figure 6). As expected, single-nucleotide (1-bp) indels were the most common, accounting for approximately 38\% (OLEI vs. RETI) to 54\% (ASSA vs. PUBI) of all indels. Xu et al. [21] concluded that 1-3 bp indels were mainly attributed to the SSR polymorphisms. Yamane et al. [45] and McCluskey et al. [46] observed that the number of indels decreased rapidly with the increase of indel lengths. However, we observed that the 5-6-bp indels were the almost second most abundant of all characterized indels, except for OLEI, rather than 2-bp indels, and the number of 5-6-bp indels was apparently more than that of 3-4-bp indels. It is likely that such 5-6-bp indels were caused by adjacent 5-6-bp motif duplications or losses, making it the second most common type [21].

Table 5 The numbers and ratios of nucleotide substitutions and indels in the five Camellia chloroplast genomes

\begin{tabular}{llllll}
\hline Genomes & ASSA & OLEI & PUBI & PETE & RETI \\
\hline ASSA & $/$ & 53 & 28 & 57 & 69 \\
OLEI & $265(5.00)$ & $/$ & 61 & 45 & 65 \\
PUBI & $82(2.93)$ & $252(4.13)$ & $/$ & 65 & 72 \\
PETE & $217(3.81)$ & $204(4.53)$ & $193(2.97)$ & $/$ & 64 \\
RETI & $242(3.51)$ & $224(3.45)$ & $221(3.07)$ & $171(2.67)$ & $/$ \\
\hline
\end{tabular}

The upper triangle shows the number of indels, while the lower triangle indicates the total nucleotide substitutions. The ratios of nucleotide substitutions to indels (S/I) are given in brackets.

\section{Molecular marker identification}

Molecular evolutionary rates are often associated with life history in flowering plants [47]. The Camellia species with rather long-generation times may have evolved slowly. Considering that a low rate of molecular evolution could complicate the phylogenetic analysis of Camellia, the identification of rapidly evolving $\mathrm{cp}$ genomic regions is critical through comparative genomic analysis. For purposes of the subsequent evolutionary and phylogenetic analyses, whole cp genome annotation and sequence comparisons showed that the number and distribution patterns of variable characters in coding and non-coding regions were fairly different among the five Camellia cp genomes (Figure 7). Among them, the proportions of variability in non-coding regions ranged from 0 to $44.4 \%$ with a mean value of $1.79 \%$, which were twice as much as in the coding regions ( $0.72 \%$ on average). Fewer mutations were observed within IR regions, including coding and non-coding regions, than LSC and SSC regions. For coding regions, the remarkably high proportions of variability of rps19 were observed in all five Camellia cp genomes. The high variability of gene rps19 might result from their extension into IR region where intrachromosomal recombinations frequently occurred to ensure the stability and consistency of IRs [25]. The proportion of variability in Camellia was lower than that in grasses [28]. We thus chose the 15 most variable non-coding regions that may serve as candidate markers for phylogenetic reconstruction (Additional file 6: Table S5), which were identified with variations that exceed $1.5 \%$ in the five Camellia cp genomes. They were $\operatorname{trnH}$-GUG/psbA, psbK/psbI, trnS-GCU/trnG-GCC, trnG-GCC intron, atpF/atpH, trnE-UUC/trnT-GGU, trnSUGA/psbZ, psaA/ycf3, trnP-UGG/psaJ, trnT-UGU/trnLUAA, rps18/rpl20, petD/rpoA, ycf15/trnL-CAA, ndhF/ $r p l 32$ and $\operatorname{css} A / n d h D$. Two of them were located in SSC region (ndhF/rpl32, and ccsA/ndhD) and the $y c f 15 / \operatorname{trnL}$ $C A A$ was located in IR regions. However, the determination whether these 15 regions could be applied to phylogenetic analyses in Camellia requires further studies.

\section{Structural constraints on evolutionary divergence}

Previous studies suggested that evolutionary differences in the $\mathrm{cp}$ genomes are dependent on the sequence and gene functions [37] and related to the structural constraints $[37,48]$. An alignment was performed among the CDS, introns, and IGS regions, along with positional information of the five Camellia cp genomes. The sequence divergence ratios among the three regions (CDS:intron:IGS) were 1:1.1:1.9 (ASSA: four other species), 1:1.2:2.4 (OLEI: four other species), 1:1:2.6 (PUBI: four other species), 1:1.3:2.2 (PETE: four other species) and 1:1.2:2.3 (RETI: four other species), respectively (Figure 8). The result clearly suggests that the intron sequences 


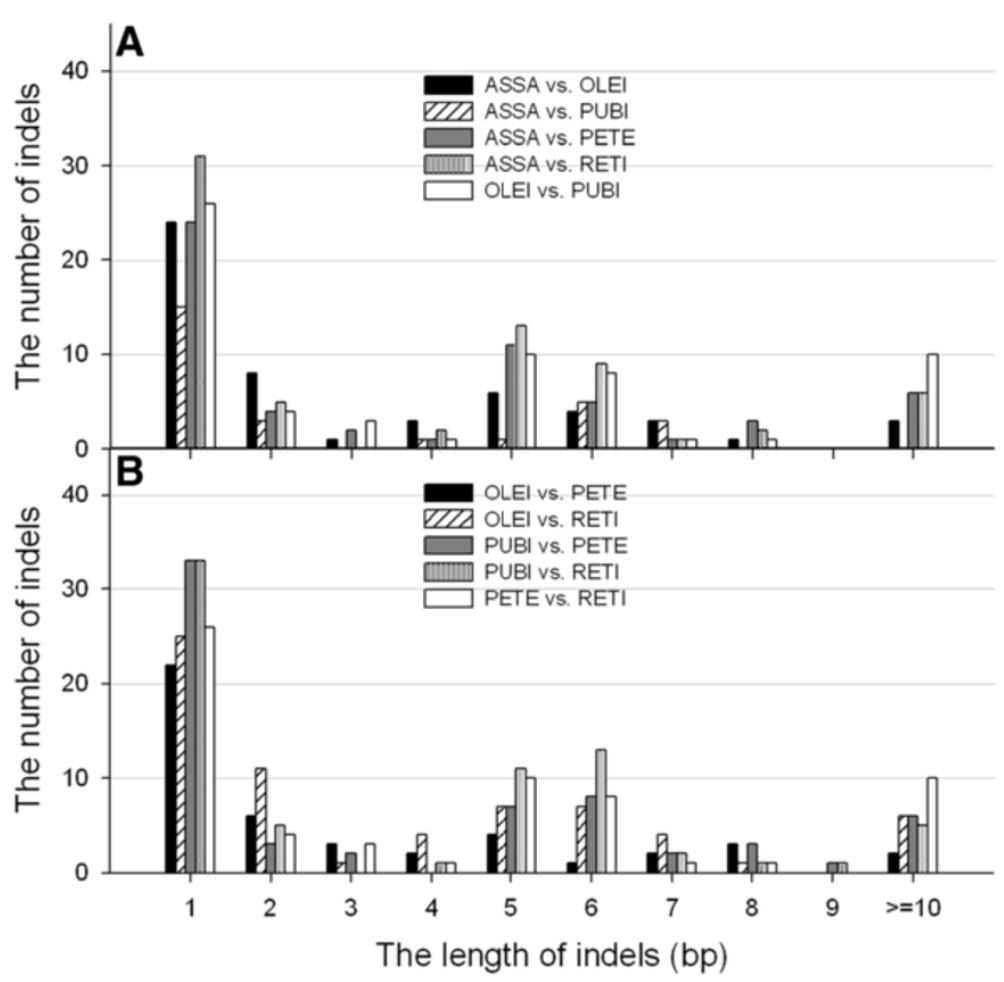

Figure 6 The distribution of indel types in the five Camellia chloroplast genomes. The pairwise comparisons were performed to identify indels among the five Camellia chloroplast genomes. A includes ASSA vs. OLEI/PUBI/PETE/RETI and OLEI vs. PUBI. B includes OLEI vs. PETE/RETI, PUBI vs. PETE/RETI and PETE vs. RETI.

have evolved faster than the CDS but slower than the IGS sequences. The rapid evolution of intron sequence was attributed to sequence divergence ratios in LSC and SSC (Additional file 7: Figure S2). This finding is also supported by a previous statement that the nucleotide substitution rates in the IGS sequences and introns are higher than the CDS [49]. The sequence alignment data sets were further partitioned into IR, LSC and SSC regions, and the sequence divergence ratios among the three regions (IR:LSC:SSC) were found to be 1:2.9:3.2 (ASSA: four other species), 1:4.1:3.9 (OLEI: four other species), 1:2.6:2.7 (PUBI: four other species) , 1:3.2:3.3 (PETE: four other species) and 1:3.1:3.0 (RETI: four other species), respectively (Figure 8 ). Such comparisons apparently indicate that the IR regions may have evolved much more slowly than the LSC and SSC regions, and levels of evolutionary divergence of introns in IRs were much lower than those of introns in LSCs and SSCs (Additional file 7: Figure S2). The frequent intrachromosomal recombination events between these two identical IR regions of the cp genome provide selective constraints on both sequence homogeneity and structural stability [25,50]. This could be used to explain why the IR regions exhibit slow nucleotide substitution rates in comparison with the SSC and LSC regions in this study. Our results thus confirm that positional effects are stronger constraints for sequence evolution than the functional groups of chloroplast genes, in good agreement with the previous observation [37].

\section{Phylogenomic analyses}

The phylogenetic studies based on the cp genome sequences are addressed successfully for the phylogenetic issues of angiosperm $[12,28,51]$. The species in the sect. Thea have long been considered to be a complex and taxonomically difficult group because of their alike morphological characteristics. Chang et al. [52] classified the sect. Thea into a total of 4 series, which comprised 42 species and four varieties. However, Min et al. [2] proposed to taxonomically classify this section into 12 species and 6 varieties. In particular, there is a controversy on the taxonomy of C. pubicosta native to Laos. This species was classified into sect. Thea by Chang et al. [52], while Min et al. [53] insisted to classify into sect. Corallina considering that some characters of C. pubicosta are different with that of other members in sect. Thea. Using C. reticulata that belongs to sect. Camellia as outgroup, our phylogenetic analysis of orthologous sequences from the sampled species in this study and recently sequenced species (C. taliensis 7) of sect. Thea showed that the ML tree was mostly consistent with MP tree with high bootstrap supports, except for the position of $C$. 

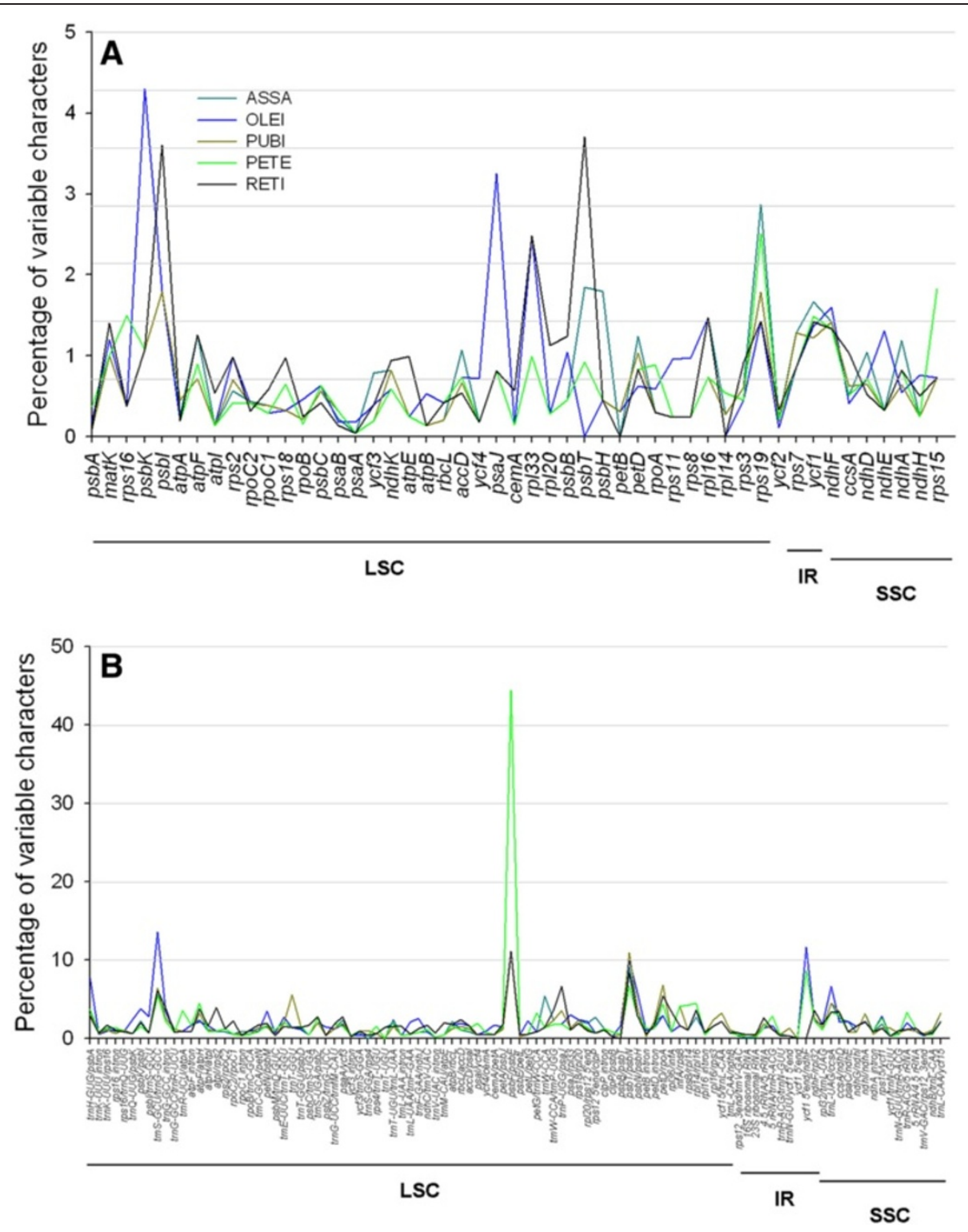

Figure 7 Percentages of variable characters in homologous regions across the five Camellia chloroplast genomes. A Coding regions; B Non-coding regions.

taliensis (Figure 9). It is notable that C. pubicosta was sister to C. sinensis var. assamica and C. grandibracteata with $\mathrm{BS}=100 \%$ (Figure 9A and B), supporting Chang's taxonomical treatment that C. pubicosta was classified into sect. Thea. Previous phylogenetic analysis using RAPDs reported that the species of sect. Thea could be divided into two groups, consistent with the number of locule ovary, that is, 5-locule ovary group and 3-locule ovary group $[5,54]$. As the first well-supported phylogenomic analyses of sect. Thea, however, our results evidently demonstrated that phylogenetic relationships and molecular evolution of the species in sect. Thea did not well follow the number of locule ovary. For example, C. fangchengensis, C. ptilophylla, C. tachangensis, $C$. kwangsiensis and C. crassicolumna var. crassicolumna were well supported as monophyletic. However, C. fangchengensis and C. ptilophylla belonged to Ser. Sinenses with 3 ovaries, while C. tachangensis, C. kwangsiensis and C. crassicolumna var. crassicolumna were members of Ser. Quinquelocularis and Ser. pentastylae with 5 ovaries. Our results thus indicated that taxonomical value of the number of ovary may be reconsidered to classify the Camellia species.

The phylogenetic analyses were performed based on the entire cp genome sequences from 18 Camellia cp genomes (Figure 9C and D), showing that the species of sect.Thea formed a monophyletic clade, except for the three 


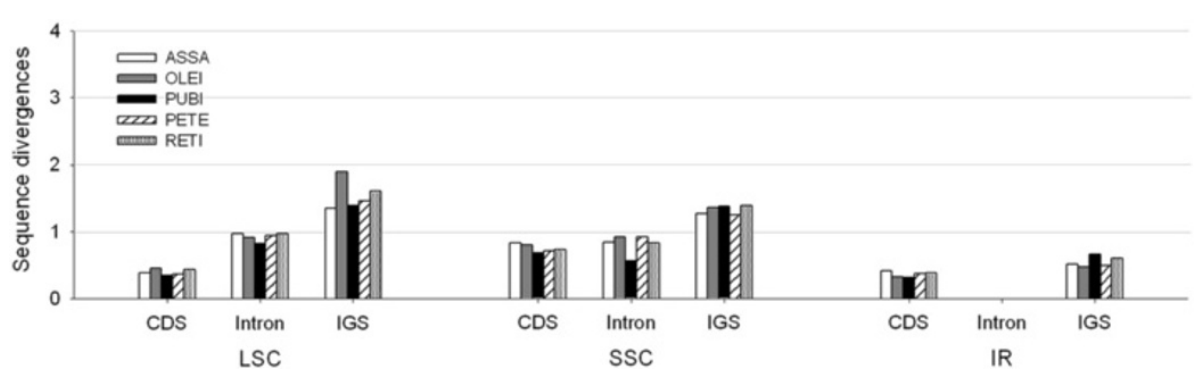

Figure 8 Levels of evolutionary divergences among different regions of the five Camellia chloroplast genomes.

individuals of C. taliensis, which is close to C. yunnanensis. This result indicated that $C$. taliensis may not be the ancestors of $C$. sinensis var. assamica [16] and there might be hybridization between $C$. taliensis and $C$. yunnanensis due to chimeric habitats. We observed that C.danzaiensis, C.pitardii and C.reticulata formed a monophyletic clade with strong bootstrap support, which might suggest that $C$. danzaiensis belongs to Subgen. Camellia, rather than Subgen. Thea. The different positions of $C$. impressinervis and C. cuspidate in ML and MP tree made more samples to resolve their phylogenetic relationship is essential. Further genomic and taxon sampling and more complete cp genomes of Camellia are deserved in further studies as phylogenomic analysis tends to suffer from the poor sampling [55].

Indels not only play an important role in elucidating genome evolution $[20,42]$, but also have potential value in constructing phylogenies [56,57]. A total of 63 putative informative indels were identified by pairwise comparisons, and then mapped to the cp genome-based phylogenetic tree using $C$. arabic as outgroup (Figure 10). Of these, 46, 13 and 4 indels were located in introns, CDS and CDS-IGS, respectively. Among these five branches, the branch resulting in RETI contained the most number of indels (17 of 63). We observed the most number of indels that were shared between
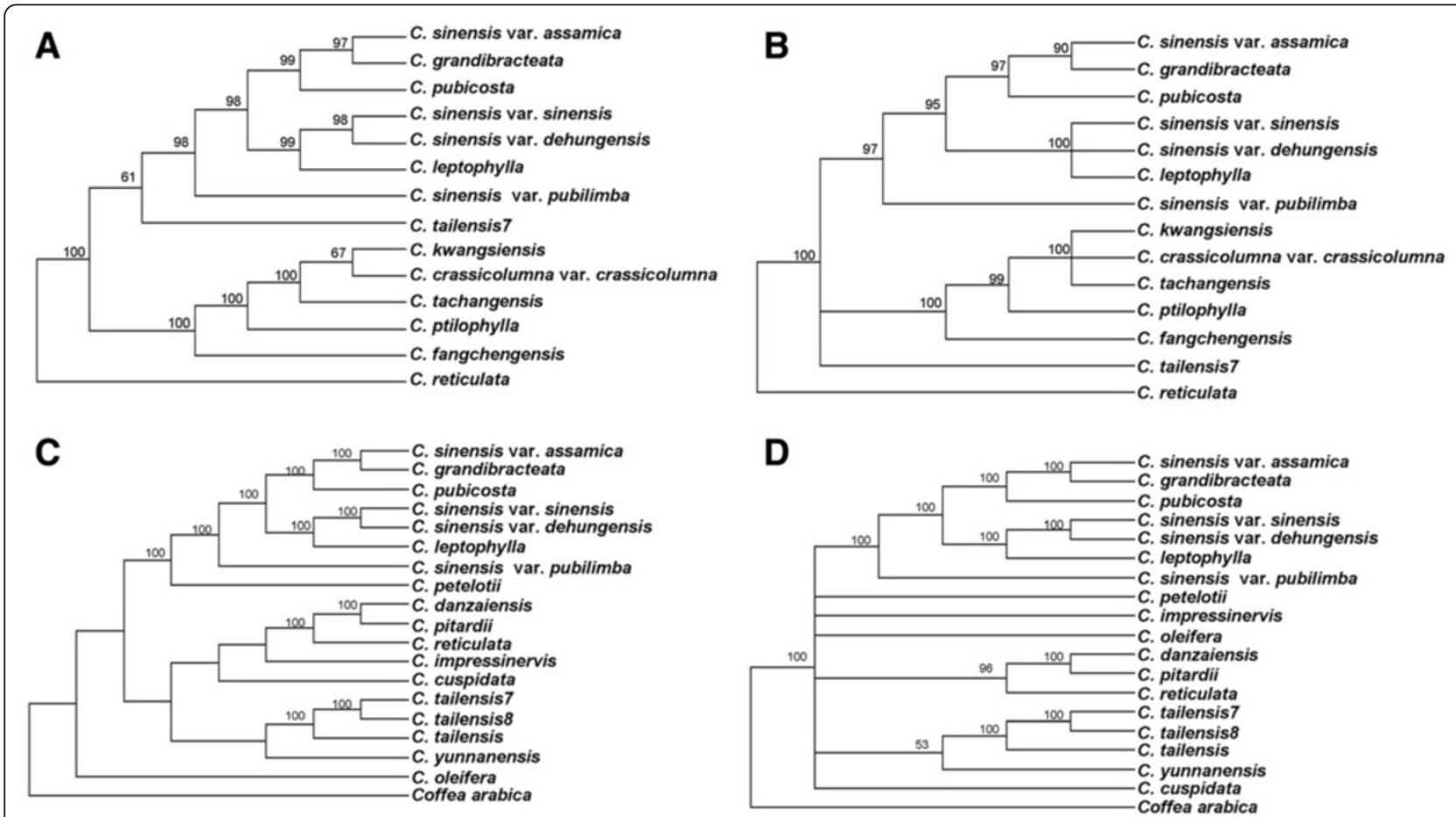

Figure 9 Phylogenetic relationships of the thirteen species of section Thea and the eighteen species of Camellia constructed by maximum likelihood $(A, C)$ and maximum parsimony $(B, D)$ with $C$. reticulata and Coffea arabica as outgroup. The $A$ and $C M L$ trees have a $-\mathrm{InL}=124830.0859$ and $-\mathrm{InL}=290325.4563$. The B MP tree has a length of 1,129 with a consistency index of 0.890 and a retention index of 0.766 . The D MP tree has a length of 14,892 with a consistency index of 0.983 and a retention index of 0.796 . Numbers above node are bootstrap support values (>50\%). 


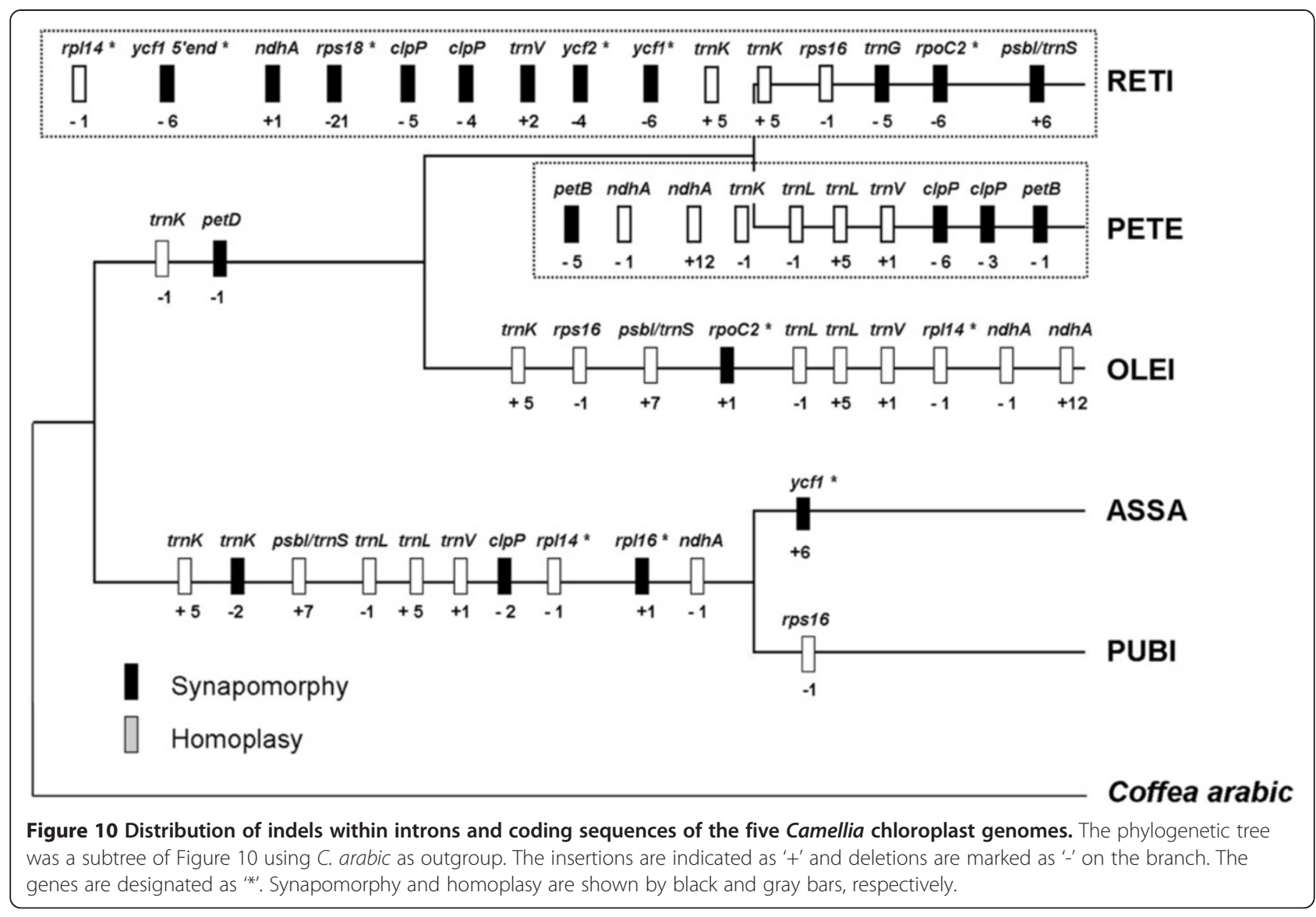

ASSA (10/11) and PUBI (10/11), suggesting their short divergence times and close relationships. Of all indels, 26 were able to be mapped to phylogenetic tree with high bootstrap supports and thus are indicative of synapomorphies. The remaining 37 indels may be homoplasies possibly associated with parallel mutations or back mutations during evolutionary history, which somehow had negative effects on the reconstruction of phylogenetic tree. Such indels should be carefully used especially when a few number of DNA fragments were applied for phylogenetic studies.

\section{Conclusions}

We reported eight complete and five draft cp genomes in the genus Camellia using Illumina sequencing technology via a combination of de novo and reference-guided assembly. These cp genomes were found highly conserved each other. We investigated the variation of repeat sequences, SSRs, indels and substitutions among the five complete Camellia cp genomes, representing a wide phylogenetic diversity in the genus Camellia. The fifteen rapidly evolving regions were identified across these $\mathrm{cp}$ genomes that could serve as potential molecular markers for further phylogenetic studies. This study is undoubtedly the first successful attempt to provide well-supported evolutionary relationships of sect. Thea based on phylogenomic analyses. The obtained cp genomes may facilitate the development of biotechnological applications for these economically important woody plants, and offer useful genetic information for purposes of phylogenetics, taxonomy and species identification in the genus Camellia.

\section{Methods}

\section{Plant materials}

Leaf materials of the Camellia plants used in this study were collected from Kunming Institute of Botany (Chinese Academy of Sciences), Tea Research Institute (Yunnan Academy of Agricultural Sciences) and International Camellia Species Garden (Jinhua, Zhejiang Province, China) in May 2011 (Table 1). The collected plant materials were classified by Min's taxonomic treatment [2] (Table 1). C. gymnogyna and C. costata of sect. Thea were unavailable and thus were absent in this study.

\section{DNA sequencing and genome assembly}

Approximately $20 \mathrm{~g}$ of fresh leaves from each species were harvested for cpDNA isolation using an improved extraction method that includes high ionic strength buffer at low $\mathrm{pH}$ (3.8) [58]. After DNA isolation, $5 \mu \mathrm{g}$ of purified DNA was fragmented by nebulization with 
compressed nitrogen gas, and constructed short-insert (300 bp) libraries following the manufacturer's protocol (Illumina). DNA from the different species was indexed by tags and pooled together in one lane of Illumina's Genome Analyzer for sequencing $(2 \times 100 \mathrm{bp})$ at Germplasm Bank of Wild Species in Southwest China, Kunming Institution of Botany, Chinese Academy of Sciences. Raw reads were first filtered to obtain the high-quality clean data by removing adaptor sequences and low-quality reads with Q-value $\leq 20$. Then, those reads mixed non-cp DNA from the nucleus and mitochondria were isolated based on the known cp genome sequences. Then, the following three steps were used to assemble cp genomes [19]. First, the filtered reads were assembled into contigs using SOAPdenovo [59]. Second, contigs were aligned to the reference genome of $C$. sinensis var. assamica (Genbank ID: JQ975030) using BLAST, and aligned contigs ( $\geq 90 \%$ similarity and query coverage) were ordered according to the reference genome. Third, raw reads were again mapped to the assembled draft cp genomes that were then visualized by Geneious (version 5.1) [60], and the majority of gaps were filled through local assembly.

Based on the reference genome of $C$. sinensis var. assamcia, we designed four primer pairs for the verification of the four junctions between the single-copy segments and IRs (as given in Additional file 1: Table S1), respectively. PCR products were then sequenced following standard Sanger protocols on ABI $3730 \times 1$ instruments. Sanger sequences and assembled genomes were aligned using Geneious assembly software to determine if there were any differences.

\section{Genome annotation, alignment and visualization}

The chloroplast genes were annotated using an online DOGMA tool [61], using default parameters to predict protein-coding genes, transfer RNA (tRNA) genes, and ribosome RNA (rRNA) genes. Start and stop codons of protein-coding genes were searched and determined by BLASTX against the NCBI protein database, with $C$. sinensis var. assamica as a guide. Genome maps were drawn with OGDraw (version 1.2) [62]. Multiple alignments were made using MAFFT version 5 [63] and adjusted manually where necessary. Full alignments with annotations were visualized using the VISTA viewer [24].

\section{Characterization of repeat sequences and SSRs}

REPuter [26] was used to identify and locate the repeat sequences, including direct, reverse and palindromic repeats within $\mathrm{cp}$ genome. For repeat identification, the following constraints were set to REPuter: (i) minimum repeat size of $30 \mathrm{bp}$, and (ii) $90 \%$ or greater sequence identity, based on Hamming distance of 3 .
SSRs were predicted using MISA [64] with the parameters set to ten repeat units $\geq 10$ for mononucleotide SSRs, six repeat units $\geq 6$ for dinucleotide, five repeat units $\geq 5$ for trinucleotide, four repeat units $\geq 4$ for tetranucleotide, and three repeat units $\geq 3$ for pentanucleotide and hexanucleotide SSRs.

\section{Identification of molecular markers}

To identify the divergent regions for phylogenetic analyses, all the regions, including CDS, introns and IGS from the Camellia cp genomes, were sequentially extracted. For each species, homologous regions of cp genomes were aligned using MAFFT version 5 and manual adjustments were made where necessary. Subsequently, the percentage of variable characters for each region was obtained. The proportion of mutational events (or variation\%) was calculated by following the modified version of the formula used in Gielly and Taberlet [65]. The proportion of mutation events $=[(\mathrm{NS}+\mathrm{ID}) / \mathrm{L}] \times 100$, where $\mathrm{NS}=$ the number of nucleotide substitutions, ID $=$ the number of indels, $\mathrm{L}=$ the aligned sequence length.

\section{Phylogenetic analysis}

The Camellia cp genome sequences were aligned using the program MAFFT version 5 [63] and adjusted manually where necessary. The ambiguously aligned loci (e.g., 'N') were excluded from the analyses. The unambiguously aligned DNA sequences were used for the reconstruction of phylogenetic trees. The phylogenetic analyses were performed based on the following two data sets: (1) the remaining sequences with lengths from 83,585 to 83,835 bp (including $78.1 \%$ coding and $21.9 \%$ non-coding regions) after the removal of the 'N's in incomplete cp genomes as well as the corresponding orthologous sequences in complete cp genomes from the alignment of the 13 Camellia cp genomes that belong to sect. Thea with C. reticulata as outgroup; (2) the eight complete cp DNA sequences sequenced obtained in this study, three Camellia cp genomes adopted from [18], and seven Camellia cp genomes retrieved from [9] with C. arabica as outgroup.

ML analyses were implemented in RAxML version 7.2.6 [66]. RAxML searches relied on the general time reversible (GTR) model of nucleotide substitution with the gamma model of rate heterogeneity. Non-parametric bootstrapping used 1,000 replicates as implemented in the "fast bootstrap" algorithm of RAxML. MP analyses were performed with PAUP*4.0b10. Heuristic tree searches were conducted with 1,000 random-taxon-addition replicates and tree bisection-reconnection (TBR) branch swapping, with "multrees" option in effect. Non-parametric bootstrap analysis was conducted under 1,000 replicates with TBR branch swapping. 


\section{Availability of supporting data}

These cp genomes sequenced in this study are available the GenBank database under the accession numbers (KJ806274-KJ806286). The alignments and phylogenetic trees supporting the results of this article are available in the TreeBASE repository, http://purl.org/phylo/treebase/ phylows/study/TB2:S16027?x-accesscode=e9a8a916b74d33 2d14f1954ca00a51f6\&format=html.

\section{Additional files}

Additional file 1: Table S1. Primers used for junction verification.

Additional file 2: Table S2. The list of accession numbers of the chloroplast genome sequences reported in this study.

Additional file 3: Figure S1. Phylogenetic tree of the fourteen sections in the genus Camellia. The indicated phylogenetic relationships of the genus were constructed by using morphological data and adopted from Min et al. [2]. The arrowheads indicated that the species (right) was classified into the section (left). The two subgenera recognized in Camellia are given on the right side of the figure.

Additional file 4: Table S3. Repeat sequences identified in the five Camellia chloroplast genomes.

Additional file 5: Table S4. SSRs characterized in the five Camellia chloroplast genomes.

Additional file 6: Table S5. The information of candidate markers with the variations more than $1.5 \%$.

Additional file 7: Figure S2. The variable characters of the seventeen intron regions of the five Camellia chloroplast genomes.

\section{Abbreviations}

AFLPs: Amplified fragment length polymorphisms; ASSA: C. sinensis var. assamica; BS value: Bootstrap support values; CDS: Coding sequences: CNS: Conserved noncoding sequences; cp: Chloroplast; IR: Inverted repeat; ISSR: Inter-simple sequence repeat; ITS: Internal transcribed spacer; IGS: Intergenic spacer; LSC: Large single copy; ML: Maximum likelihood; MP: Maximum parsimony; OLEl: C. oleifera; PETE: C. petelotii; PUBI: C. pubicosta; RAPD: Random amplified polymorphic DNA; RETI: C. reticulata; rRNA: Ribosomal RNA; S/I: Nucleotide substitutions events to indels events; SSC: Small single copy; SSRs: Simple sequence repeats; TBR: Tree bisection-reconnection; tRNA: Transfer RNA.

\section{Competing interests}

The authors declare that they have no competing interests.

\section{Authors' contributions}

Conceived and designed the experiments: LZG. Performed the experiments: $\mathrm{HH}$ and SYM. Analyzed the data: HH, CS and YL. Contributed reagents/ materials/analysis tools: $\mathrm{HH}$. Wrote the paper: $\mathrm{LZG}$ and $\mathrm{HH}$. All authors read and approved the final manuscript.

\section{Acknowledgements}

We thank Zhu Ting, Nan Hu and Chen Qing for their help to sample the Camellia germplasms and experimental assistance, Ju Gao and Qun-Jie Zhang for data analysis, and anonymous reviewers for their valuable comments. This work was supported by National Science Foundation of China (U0936603), Top Talents Program of Yunnan Province (20080A009) and Hundreds of Oversea Talents Program of Yunnan Province to L.Z. GAO and National Science Foundation of China (31200515) and Surface Project of Natural Science Foundation of Yunnan Province (2012FB179) to H. Huang.

\section{Author details}

${ }^{1}$ Plant Germplasm and Genomics Center, Germplasm Bank of Wild Species in Southwest China, Kunming Institute of Botany, Chinese Academy of Sciences, Kunming 650201, China. ${ }^{2}$ University of the Chinese Academy of Sciences, Beijing 100039, China.
Received: 1 January 2014 Accepted: 20 June 2014

Published: 7 July 2014

\section{References}

1. Vijayan K, Zhang WJ, Tsou CH: Molecular taxonomy of Camellia (Theaceae) inferred from nrlTS sequences. Am J Bot 2009, 96:1348-1360.

2. Min TL, Bruce B: Flora of China. Beijing, China: Science Press; 2010.

3. Wachira FN, Tanaka J, Takeda Y: Genetic variation and differentiation in tea (Camellia sinensis) germplasm revealed by RAPD and AFLP variation. J Hort Sci Biotech 2001, 76:557-563.

4. Wang LY, Liu BY, Jiang YY, Duan YS, Cheng H, Zhou J, Tang YC: Phylogenetic analysis of inter species in section Thea through SSR markers. J Tea Sci 2009, 29:341-346.

5. Chen L, Yamaguchi S, Wang PS, Xu M, Song WX, Tong QQ: Genetic polymorphism and molecular phylogeny analysis of section Thea based on RAPD markers. J Tea Sci 2002, 22:19-24.

6. Ji PZ, Wang YG, Zhang J, Tang YC, Huang XQ, Wang PS: Genetic relationships between sect. Thea from Yunnan province revealed by inter-simple sequence repeat polymerase China reaction. Southwest China J Agric Sci 2009, 22:584-588.

7. Tian M, Li JY, Ni S, Fan ZQ, Li XL: Phylogenetic study on section Camellia based on ITS sequences data. Acta Hort Sin 2008, 35:1685-1688.

8. Fang W, Yang JB, Yang SX, Li DZ: Phylogeny of Camellia sects. Longipedicellata, Chrysantha and Longissima (Theaceae) based on sequence data of four chloroplast DNA Loci. Acta Bot Yunnanica 2010, 32:1-13.

9. Yang JB, Yang SX, Li HT, Yang J, Li DZ: Comparative chloroplast genomes of Camellia species. PLOS ONE 2013, 8:e73053.

10. McCauley DE, Stevens JE, Peroni PA, Raveill JA: The spatial distribution of chloroplast DNA and allozyme polymorphisms within a population of Silene alba (Caryophyllaceae). Am J Bot 1996, 83:727-31.

11. Small RL, Cronn RC, Wendel JF: Use of nuclear genes for phylogeny reconstruction in plants. Aust Syst Bot 2004, 17:145-70.

12. Jansen RK, Cai Z, Raubeson LA, Daniell H, de Pamphilis CW, Leebens-Mack J, Müller KF, Guisinger-Bellian M, Haberle RC, Hansen AK, Chumley TW, Lee S, Peery R, McNeal JR, Kuehl J, Boore JL: Analysis of 81 genes from 64 plastid genomes resolves relationships in angiosperms and identifies genome-scale evolutionary patterns. Proc Natl Acad Sci U S A 2007, 104:19369-19374.

13. Parks M, Cronn R, Liston A: Increasing phylogenetic resolution at low taxonomic levels using massively parallel sequencing of chloroplast genomes. BMC Biol 2009, 7:84.

14. Moore MJ, Soltis PS, Bell CD, Burleigh JG, Soltis DE: Phylogenetic analysis of 83 plastid genes further resolves the early diversification of eudicots. Proc Natl Acad Sci U S A 2010, 107:4623-4628.

15. Huang H, Tong Y, Zhang QJ, Gao LZ: Genome Size Variation among and within Camellia Species by Using Flow Cytometric Analysis. PLOS ONE 2013, 8:e64981.

16. Liu Y, Yang SX, Ji PZ, Gao LZ: Phylogeography of Camellia taliensis (Theaceae) inferred from chloroplast and nuclear DNA: insights into evolutionary history and conservation. BMC Evol Biol 2012, 12:92-105.

17. Provan J, Powell W, Hollingsworth PM: Chloroplast microsatellites: new tools for studies in plant ecology and evolution. Trends Ecol Evol 2001, 16:142-147.

18. Shi C, Liu Y, Huang H, Xia EH, Zhang HB, Gao LZ: Contradiction between plastid gene transcription and function due to complex posttranscriptional splicing: an exemplary study of ycf15 function and evolution in angiosperms. PLOS ONE 2013, 8:e59620.

19. Cronn R, Liston A, Parks M, Gernandt DS, Shen RK: Multiplex sequencing of plant chloroplast genomes using Solexa sequencing-by- synthesis technology. Nucleic Acids Res 2008, 36:e122.

20. Matsuoka Y, Yamazaki Y, Ogihara Y, Tsunewaki K: Whole chloroplast genome comparison of rice, maize, and wheat: implications for chloroplast gene diversification and phylogeny of cereals. Mol Biol Evol 2002, 19:2084-2091.

21. Xu Q, Xiong GJ, Li PB, He F, Huang Y, Wang KB, Li ZH, Hua JP: Analysis of Complete Nucleotide Sequences of 12 Gossypium Chloroplast Genomes: Origin and Evolution of Allotetraploids. PLOS ONE 2012, 7:e37128.

22. Davis Jl, Soreng RJ: Migration of endpoints of two genes relative to boundaries between regions of the plastid genome in the grass family (Poaceae). Am J Bot 2010, 97:874-892. 
23. Kim KJ, Lee $\mathrm{HL}$ : Complete chloroplast genome sequences from Korean ginseng (Panax schinseng Nees) and comparative analysis of sequence evolution among 17 vascular plants. DNA Res 2004, 11:247-261.

24. Mayor C, Brudno M, Schwartz JR, Poliakov A, Rubin EM, Frazer KA, Pachter LS, Dubchak I: VISTA : visualizing global DNA sequence alignments of arbitrary length. Bioinformatics 2000, 16:1046-1047.

25. Palmer JD: Plastid chromosomes: structure and evolution. In the Molecular Biology of Plastids. Edited by Bogorad L, Vasil IK. New York: Academic Press; 1991:5-53.

26. Kurtz S, Choudhuri JV, Ohlebusch E, Schleiermacher C, Stoye J, Giegerich R: REPuter: the manifold applications of repeat analysis on a genomic scale. Nucleic Acids Res 2001, 29:4633-4642.

27. Saski C, Lee SB, Fjellheim S, Guda C, Jansen RK, Luo H, Tomkins J, Rognli OA, Daniell $\mathrm{H}$, Clarke JL: Complete chloroplast genome sequences of Hordeum vulgare, Sorghum bicolor and Agrostis stolonifera, and comparative analyses with other grass genomes. Theor App/ Genet 2007, 115:571-590.

28. Zhang YJ, Ma PF, Li DZ: High-throughput sequencing of six bamboo chloroplast genomes: phylogenetic implications for temperate woody bamboos (Poaceae: Bambusoideae). PLoS ONE 2011, 6:e20596.

29. Tangphatsornruang S, Sangsrakru D, Chanprasert J, Uthaipaisanwong P, Yoocha T, Jomchai N, Tragoonrung S: The chloroplast genome sequence of Mungbean (Vigna radiata) determined by high-throughput pyrosequencing: structural organization and phylogenetic relationship. DNA Res 2010, 17:11-22.

30. Asano T, Tsudzuki T, Takahashi S, Shimada H, Kadowaki K: Complete nucleotide sequence of the sugarcane (Saccharum officinarum) chloroplast genome: A comparative analysis of four monocot chloroplast genomes. DNA Res 2004, 11:93-99.

31. Timme RE, Kuehl JV, Boore $J \mathrm{~L}$, Jansen RK: A comparative analysis of the Lactuca and Helianthus (Asteraceae) plastid genomes: Identification of divergent regions and categorization of shared repeats. Am J Bot 2007, 94:302-312.

32. Cavalier-Smith T: Chloroplast evolution: secondary symbiogenesis and multiple losses. Curr Biol 2002, 12:62-64.

33. Gao L, Yi X, Yang YX, Su YJ, Wang T: Complete chloroplast genome sequence of a tree fern Alsophila spinulosa: insights into evolutionary changes in fern chloroplast genomes. BMC Evol Biol 2009, 9:130-144.

34. Echt CS, DeVerno LL, Anzidei M, Vendramin GG: Chloroplast microsatellites reveal population genetic diversity in red pine, Pinus resinosa Ait. Mol Ecol 1998, 7:307-316.

35. Powell W, Morgante M, Andre C, Mcnicol JW, Machray GC, Doyle JJ, Tingey SV, Rafalski JA: Hypervariable microsatellites provide a general source of polymorphic DNA markers for the chloroplast genome. Curr Biol 1995, 5:1023-1029.

36. Kuang DY, Wu H, Wang YL, Gao LM, Zhang SZ, Lu L: Complete chloroplast genome sequence of Magnolia kwangsiensis (Magnoliaceae): implication for DNA barcoding and population genetics. Genome 2011, 54:663-673.

37. Yi D-K, Kim K-J: Complete Chloroplast Genome Sequences of Important Oilseed Crop Sesamum indicum L. PLOS ONE 2012, 7:e35872.

38. Jakobsson M, Sall T, Lind-Hallden C, Hallden C: Evolution of chloroplast mononucleotide microsatellites in Arabidopsis thaliana. Theor Appl Genet 2007, 114:223-235.

39. Garris AJ, Tai TH, Coburn J, Kresovich S, McCouch S: Genetic structure and diversity in Oryza sativa L. Genetics 2005, 169:1631-1638.

40. Xu DH, Abe J, Gai JY, Shimamoto Y: Diversity of chloroplast DNA SSRs in wild and cultivated soybeans: evidence for multiple origins of cultivated soybean. Theor Appl Genet 2002, 105:645-653.

41. Leseberg CH, Duvall MR: The complete chloroplast genome of Coix lacryma-jobi and a comparative molecular evolutionary analysis of plastomes in cereals. J Mol Evol 2009, 69:311-318.

42. Grover CE, Yu Y, Wing RA, Paterson AH, Wendel JF: A phylogenetic analysis of indel dynamics in the cotton genus. Mol Biol Evol 2008, 25:1415-1428.

43. Britten RJ, Rowen L, Williams J, Cameron RA: Majority of divergence between closely related DNA samples is due to indels. Proc Natl Acad Sci U S A 2003, 100:4661-4665.

44. Chen JQ, Wu Y, Yang H, Bergelson J, Kreitman M, Tian D: Variation in the ratio of nucleotide substitution and indel rates across genomes in mammals and bacteria. Mol Biol Evol 2009, 26:1523-1531.

45. Yamane K, Yano K, Kawahara T: Pattern and rate of indel evolution inferred from whole chloroplast intergenic regions in sugarcane, maize and rice. DNA Res 2006, 13:197-204.
46. McCluskey K, Wiest AE, Grigoriev IV, Lipzen A, Martin J, Schackwitz W, Baker SE: Rediscovery by whole genome sequencing: classical mutations and genome polymorphisms in Neurospora crassa. G3 (Bethesda) 2011, 1:303-316.

47. Smith SA, Donoghue MJ: Rates of molecular evolution are linked to life history in flowering plants. Science 2008, 322:86-89.

48. Perry AS, Wolfe $\mathrm{KH}$ : Nucleotide substitution rates in legume chloroplast DNA depend on the presence of the inverted repeat. J Mol Evol 2002, 55:501-508.

49. Clegg MT, Gaut BS, Learn GH, Morton BR: Rates and patterns of chloroplast DNA evolution. Proc Natl Acad Sci U S A 1994, 91:6795-6801.

50. Wolfe KH, Gouy ML, Yang YW, Sharp PM, Li WH: Date of the monocot-dicot divergence estimated from chloroplast DNA sequence data. Proc Natl Acad Sci U S A 1989, 86:6201-6205.

51. Moore MJ, Bell CD, Soltis PS, Soltis DE: Using plastid genome-scale data to resolve enigmatic relationships among basal angiosperms. Proc Natl Acad Sci U S A 2007, 104:19363-19368.

52. Chang HD, Ren SX: Flora of China. Science Press. Tomus 1998, 49(3):1-251.

53. Min TL: A revision of Camellia sect. Thea Acta Bot Yunnanica 1992, 14:115-132.

54. Li XH, Zhang CZ, Liu CL, Shi ZP, Luo JW, Chen X: RAPD analysis of the genetic diversity in Chinese tea germplasm. Acta Hort $\operatorname{Sin} 2007$, 34:507-508.

55. Peng ZH, Lu TT, Li LB, Liu XH, Gao ZM, Hu T, Yang XW, Feng Q, Guan JP, Weng QJ, Fan DL, Zhu CR, Lu Y, Han B, Jiang ZH: Genome-wide characterization of the biggest grass, bamboo, based on 10,608 putative full-length cDNA sequences. BMC Plant Biol 2010, 10:116-129.

56. Bapteste $E$, Philippe $H$ : The potential value of indels as phylogenetic markers: position of Trichomonads as a case study. Mol Biol Evol 2002, 19:972-977.

57. Simmons MP, Ochoterena H, Carr TG: Incorporation, relative homoplasy, and effect of Gap characters in sequence -based phylogenetic analyses. Syst Biol 2001, 50:454-462.

58. Shi C, Hu N, Huang H, Gao J, Zhao Y-J, Gao LZ: An Improved Chloroplast DNA Extraction Procedure for Whole Plastid Genome Sequencing. PLOS ONE 2012, 7:e31468.

59. Li R, Zhu H, Ruan J, Qian W, Fang X, Shi Z, Li Y, Li S, Shan G, Kristiansen K, Li S, Yang H, Wang J, Wang J: De novo assembly of human genomes with massively parallel short read sequencing. Genome Res 2010, 20:265-272.

60. Drummond A, Ashton B, Buxton S, Cheung M, Cooper A, Duran C, Field M, Heled J, Kearse M, Markowitz S, Moir R, Stones-Havas S, Sturrock S, Thierer T, Wilson A: Geneious v5. 2011, 4: Available from http://www.geneious.com.

61. Wyman SK, Jansen RK, Boore JL: Automatic annotation of organellar genomes with DOGMA. Bioinformatics 2004, 20:3252-3255.

62. Lohse M, Drechsel O, Bock R: Organellar Genome DRAW (OGDRAW): a tool for the easy generation of high-quality custom graphical maps of plastid and mitochondrial genomes. Curr Genet 2007, 52:267-274.

63. Katoh $\mathrm{K}$, Kuma K, Toh H, Miyata T: MAFFT version 5: improvement in accuracy of multiple sequence alignment. Nucleic Acids Res 2005, 33:511-518

64. Thiel T, Michalek W, Varshney RK, Graner A: Exploiting EST databases for the development and characterization of gene-derived SSR-markers in barley (Hordeum vulgare L.). Theor Appl Genet 2003, 106:411-422.

65. Gielly $L$, Taberlet P: The use of chloroplast DNA to resolve plant phylogenies: noncoding versus rbcL sequences. Mol Biol Evol 1994 11:769-777.

66. Stamatakis A: RAxML-VI-HPC: maximum likelihood-based phylogenetic analyses with thousands of taxa and mixed models. Bioinformatics 2006, 22:2688-2690

doi:10.1186/1471-2148-14-151

Cite this article as: Huang et al:: Thirteen Camellia chloroplast genome sequences determined by high-throughput sequencing: genome structure and phylogenetic relationships. BMC Evolutionary Biology 2014 14:151. 